\title{
VARIACIONES GEOGRÁFICAS DE LA ESTRUCTURA Y COMPOSICIÓN DE LA VEGETACIÓN LEÑOSA EN EL LÍMITE ENTRE EL ESPINAL Y EL MONTE EN EL Noreste de la Patagonia (Argentina)
}

\author{
SILVIA S. TORRES ROBLES ${ }^{1}$, MARCELO F. ARTURI², CECILIA CONTRERAS ${ }^{3}$, \\ GUADALUPE PETER ${ }^{1,4}$ y JUAN M. ZEBERIO'1
}

\begin{abstract}
Resumen: La definición de las ecorregiones incide sobre el uso de la tierra ya que éstas son tomadas como base para la formulación de políticas de manejo y conservación. Los disturbios provocan variaciones de la vegetación entre sitios cercanos dificultando el establecimiento de límites entre regiones. Con el objetivo de analizar un gradiente geográfico en un área transicional entre el Espinal y el Monte, se caracterizaron estructura y composición de la vegetación leñosa en el noreste de la Patagonia y se evaluó en qué medida sitios cercanos con diferencias estructurales presentaban diferencias composicionales asimilables a cada ecorregión. La variación de la composición florística presentó cambios más importantes en dirección E-O que en dirección N-S, reflejando un amplio gradiente de transición Espinal-Monte. Los cambios estructurales más claros fueron la disminución de la altura y cobertura arbórea en oposición a la cobertura arbustiva. Tales cambios resultaron menos asociados con la variación geográfica que los cambios composicionales, exhibiendo mayor variación en dirección N-S y en menor medida en la dirección E-O. Los disturbios promueven cambios más notables en la estructura que en la composición florística por lo que resulta poco probable que provoquen un corrimiento de límites geográficos entre las ecorregiones Monte y Espinal.
\end{abstract}

Palabras clave: Límite fitogeográfico, conservación, ecorregiones, NE de Río Negro, SO de Buenos Aires.

Summary: Geographical variations in structure and composition of woody vegetation in the espinal and monte boundary in Northeastern Patagonia (Argentina). The definition of ecoregions has implications on land use, because these classifications are the basis for the formulation of management and conservation policies. Disturbances promote variations among neighboring sites, and difficult the establishment of boundaries between ecoregions. With the aim of analyzing a geographical gradient in a transitional area between Espinal and Monte regions, we characterized the structure and composition of woody vegetation in the northeastern Patagonia, and we evaluated how neighboring sites with structural differences presented compositional differences assigned to each ecoregion. Variation in floristic composition presented more important changes in E-W direction than in N-S direction, reflecting a broad transitional gradient between Espinal and Monte. The most remarkable structural changes were the decrease in height and cover of trees in opposition to shrub cover. These changes were less associated with geographical variation than compositional changes, exhibiting a more evident trend of variation in $\mathrm{N}-\mathrm{S}$ direction than in $\mathrm{E}-\mathrm{W}$ direction. Disturbances promote more notable changes in structure than in floristic composition, then it is less probable that they may cause a disruption in the geographical boundaries between Espinal and Monte ecoregions.

Key words: Phytogeographical boundaries; conservation, ecoregion, NE Rio Negro province, SW Buenos Aires province.

\footnotetext{
1 Sede Atlántica, Universidad Nacional de Río Negro. Av. Don Bosco y Av. Leloir, 8500, Viedma, Río Negro, storresr@ unrn.edu.ar.

2 LISEA, Facultad de Ciencias Agrarias y Forestales, UNLP. Diagonal 113 № 469, 1900, La Plata, Buenos Aires, talares@ agro.unlp.edu.ar.

${ }^{3}$ Facultad de Ciencias Agrarias y Forestales, UNLP, 60 y 119, 1900, La Plata, Buenos Aires.

${ }^{4}$ CONICET.
} 


\section{INTRODUCCIÓN}

La definición de las ecorregiones puede incidir directamente sobre el uso de la tierra ya que en muchos casos dichas áreas son tomadas como base para la formulación de políticas de manejo y conservación. La homogeneidad de la vegetación dentro de las ecorregiones es una de las condiciones necesarias para su delimitación, tanto por su capacidad de reflejar cambios ambientales como por su influencia sobre las comunidades animales, entre otros aspectos relevantes. Por ese motivo, los cambios en la composición y estructura de la vegetación asociadas a variaciones geográficas, el clima, la topografía y el suelo, conllevan al establecimiento de límites entre ecorregiones (Cabrera, 1976; Burkart et al., 1999; Brown et al., 2006; Morello et al., 2012). Cuando el cambio espacial de la estructura y composición de la vegetación es abrupto, el establecimiento de límites resulta más sencillo que cuando ocurren gradientes espaciales suaves (Hernández Bermejo \& Sainz Ollero, 1984). Además, la definición de esquemas biogeográficos se ve dificultada por el dinamismo de las comunidades vegetales en respuesta a disturbios naturales o antrópicos (Arturi, 2006). Una situación en la que estas dificultades podrían manifestarse es el límite sur del Espinal, en su contacto con el Monte, en el NE de la Patagonia. En trabajos clásicos de fitogeografía (Cabrera, 1976; Cabrera \& Willink, 1980) y revisiones más recientes (Burkart et al., 1999; Brown et al., 2006; Morello et al., 2012) el límite sur del Espinal con el Monte se establece al sur del Río Colorado, incluyendo al E del departamento Pichi Mahuida en la provincia de Río Negro y al partido de Patagones en la provincia de Buenos Aires como parte del distrito del Caldén de la provincia fitogeográfica del Espinal. Morello et al. (2012), si bien también consideran a esta región como parte del distrito del Caldén, indican que los límites de este distrito son difíciles de establecer porque hacia el $\mathrm{O}$ forma extensos ecotonos con las ecorregiones Monte y Pampa, donde se entremezclan las especies de ambas regiones con la influencia de disturbios naturales o antrópicos. Las actividades humanas pueden provocar diferencias estructurales importantes entre sitios cercanos. La respuesta de la vegetación a esos disturbios podría promover diferencias florísticas, fisonómicas y estructurales.
En este trabajo se caracterizó la estructura y composición de la vegetación leñosa en el NE de la provincia de Río Negro y SO de la provincia de Buenos Aires con la finalidad de analizar el gradiente geográfico de la estructura y composición en un área de transición entre el Espinal y el Monte. Se evaluó en qué medida sitios cercanos con diferencias estructurales, probablemente provocadas por actividades humanas, presentaban diferencias de composición asimilables a las diferentes ecorregiones: sitios con mayor desarrollo estructural asociados al Espinal y sitios con escaso desarrollo estructural, al Monte.

\section{Materiales y Métodos}

\section{Área de estudio}

El trabajo se llevó a cabo en el NE de la Patagonia, incluyendo el sector SO de Buenos Aires (partidos de Villarino y Patagones), NE de Río Negro (departamentos Pichi Mahuida, General Conesa y Adolfo Alsina) (Fig.1). La fisonomía varía desde el bosque xerófilo hasta el matorral o estepa arbustiva xerófila (Fosberg, 1961). El clima del área de estudio es subtemplado seco de transición, con veranos cálidos e inviernos moderados, sin ningún exceso estacional de agua, ventoso especialmente en primavera y verano, lo cual contribuye a hacer más marcado el déficit de humedad del suelo. Las precipitaciones varían en un gradiente SO-NE (Fig. 1), aproximadamente de 300 a $590 \mathrm{~mm}$ anuales y con máximos en otoño y primavera presentando una alta variabilidad entre años (Godagnone \& Bran, 2009).

\section{Muestreo}

Se establecieron 28 puntos de muestreo distribuidos en los partidos y departamentos provinciales cubriendo un área de aproximadamente $250 \mathrm{~km}$ en dirección latitudinal y $140 \mathrm{~km}$ en dirección longitudinal (Fig. 1). Como consecuencia de variaciones ambientales y el efecto de las actividades humanas la vegetación leñosa se distribuye en manchas de tamaño y estado de conservación variables. Por ese motivo, para determinar la localización de cada sitio se llevó a cabo una interpretación visual de imágenes satelitales de uso libre (Google Earth $\left.{ }^{\circledR}\right)$ con la finalidad de detectar diferencias en la cobertura 
de la vegetación leñosa, que fueron corroboradas en el terreno. A partir de esa diferenciación se seleccionaron sitios de muestreo con cobertura de leñosas contrastantes dentro de cada partido.

Variación de la composición florística y estructural

En cada sitio de muestreo se establecieron cinco parcelas de 10 x $10 \mathrm{~m}$. Se consideraron especies arbóreas a aquellas que, ya sea en la zona de estudio o fuera de ella, pudieran alcanzar los $10 \mathrm{~cm}$ de diámetro del fuste a $1,3 \mathrm{~m}$ de altura (DAP). El resto de las especies leñosas fueron consideradas arbustivas. Para las especies arbóreas se registró el número de individuos, la altura y el DAP de los individuos mayores de $1 \mathrm{~cm}$ de DAP. La altura se midió con clinómetro y el DAP con cinta diamétrica. Además se midió el ancho máximo de la copa y la medida perpendicular al mismo para calcular la cobertura (Mateucci \& Colma, 1982) como el área de un círculo con diámetro igual al promedio de las medidas de la copa registradas. Para las especies arbustivas se determinó la cobertura y altura de individuos o grupos multiespecíficos de individuos registrándose las especies presentes. La altura se determinó con cinta métrica y la cobertura se calculó como la superficie de un círculo cuyo diámetro fue el promedio del ancho máximo de la copa del arbusto, o grupo de arbustos, y la medida perpendicular al mismo.

Sobre la base de las mediciones realizadas se calculó para cada sitio la densidad, altura promedio y cobertura de árboles, cobertura y altura promedio de arbustos, y riqueza de especies leñosas como el número total de especies arbóreas y arbustivas registradas en las cinco parcelas. Además se calculó la frecuencia de cada especie en cada sitio como la proporción de parcelas en las que se registró su presencia (Mateucci \& Colma, 1982).

Los nombres científicos de las especies y taxa infraespecíficos aceptados siguieron la nomenclatura de la Flora Argentina (Anton \& Zuloaga, 2014).

\section{Análisis de datos}

Se llevaron a cabo análisis de componentes principales (ACP) con la finalidad de analizar la variación de la composición específica y de la estructura entre sitios (Digby \& Kempton, 1987). El análisis de la composición estuvo basado en la frecuencia por especie pero sólo se incluyeron aquellas que se registraron en al menos 3 de los 28

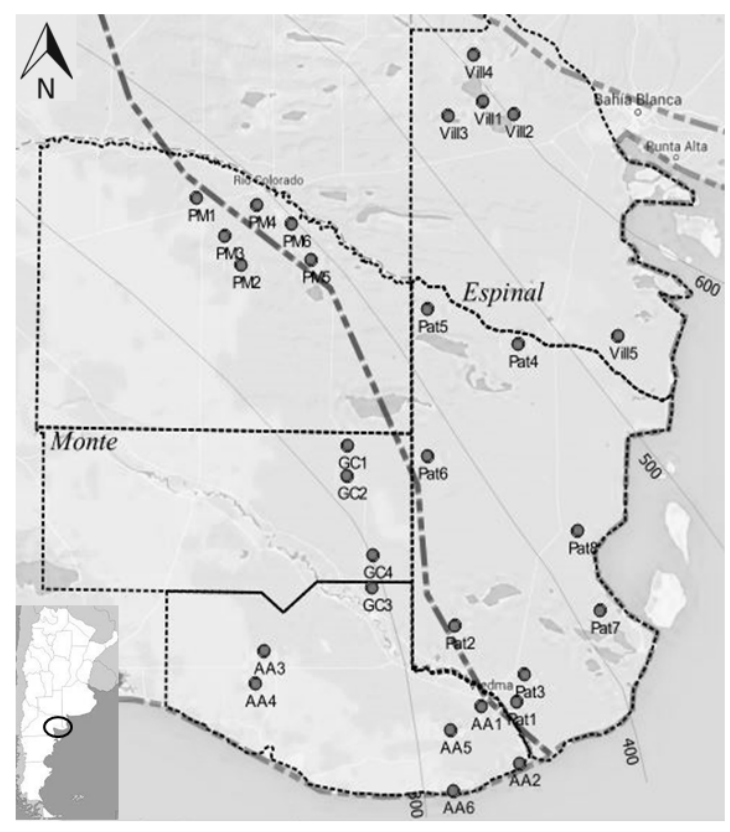

Fig. 1. Ubicación geográfica de los sitios muestreados en el área de estudio. Se indica en línea de puntos los límites de los departamentos de la provincia de Río Negro: AA: Adolfo Alsina; GC: General Conesa; PM: Pichi Mahuida; y los partidos de la provincia de Buenos Aires: VILL: Villarino; PAT: Patagones. Se indican también en línea continua las isohietas, y en linea de puntos y líneas el límite de las ecorregiones Monte y Espinal establecido por Morello et al., 2012.

sitios y sus valores fueron transformados por la raíz cuadrada para aproximar a una distribución normal y homogeneizar sus varianzas, debido a que la distribución esperada de los datos expresados en frecuencia es binomial (Digby \& Kempton, 1987). El análisis de las variables estructurales de la vegetación por sitio (cobertura de árboles, cobertura de arbustos, densidad de árboles, altura de arbustos, altura de árboles y riqueza), estuvo basado en la matriz de correlación debido a las diferencias en las unidades de las variables (Digby \& Kempton, 1987). En ambos casos se calcularon coeficientes de correlación por rangos de Spearman entre la posición de los sitios sobre los ejes del ACP y la posición latitudinal y longitudinal, previa proyección de las coordenadas geográficas de los sitios de muestreo a Gauss-Kruger. Estas correlaciones permitieron evaluar tendencias geográficas de la variación composicional y estructural. 
Se utilizó la prueba de Mantel para determinar la medida en que las diferencias composicionales entre sitios se relacionaron con diferencias estructurales. Además, mediante la prueba de Mantel Parcial, se determinó si tales diferencias persistían después de haber removido el efecto de la distancia geográfica sobre la distancia composicional. Todos los análisis se realizaron con el programa R (R Development Core Team, 2009).

\section{Resultados}

Variación de la composición florística

Las diferencias de composición florística reflejadas por el eje 1 del ACP se asociaron con la longitud $(r=0,77 ; P<0,01)$ reflejando un gradiente E-O desde el extremo negativo hacia el positivo (Fig. 2 A). Las especies que más claramente tendieron a aumentar en frecuencia hacia el extremo positivo fueron Senna aphylla (Cav.) H.S. Irwin \& Barneby, Acantholippia seriphioides (A. Gray) Moldenke, Larrea divaricata Cav., Prosopidastrum globosum (Gillies ex Hook. \& Arn.) Burkart, Prosopis flexuosa DC. var. flexuosa Phil. y Monttea aphylla (Miers) Benth. \& Hook. var. aphylla. Hacia el extremo negativo lo hicieron Geoffroea decorticans (Gillies ex Hook. \& Arn.) Burkart y Prosopis caldenia Burkart (Fig. 2 A; Tabla 1). El eje 2 del ACP se asoció con la latitud $(r=-0,66 ; P<0,01)$ reflejando un gradiente S-N desde su extremo negativo hacia el positivo. Las especies que tendieron a aumentar hacia el extremo positivo fueron $P$. flexuosa var. flexuosa y $P$. caldenia, mientras que hacia el extremo negativo lo hicieron Prosopis flexuosa DC. var. depressa F. A. Roig., Schinus johnstonii F. A. Barkley, Cyclolepis genistoides D. Don y Chuquiraga erinacea D. Don subsp. erinacea (Fig. 2 A; Tabla 1). No se hallaron correlaciones significativas entre las coordenadas geográficas y el tercer eje de ordenamiento.

Entre las especies de mayor frecuencia que no exhibieron tendencias de cambio con los dos primeros ejes de ordenamiento se encontraron Condalia microphylla Cav., Lycium chilense Miers ex Bertero y Ephedra triandra Tul. emend. J. H. Hunz (Tabla 1).

\section{Variación estructural}

El primer eje de ordenamiento de los sitios por variables estructurales reflejó un gradiente creciente en la densidad de árboles así como mayor cobertura
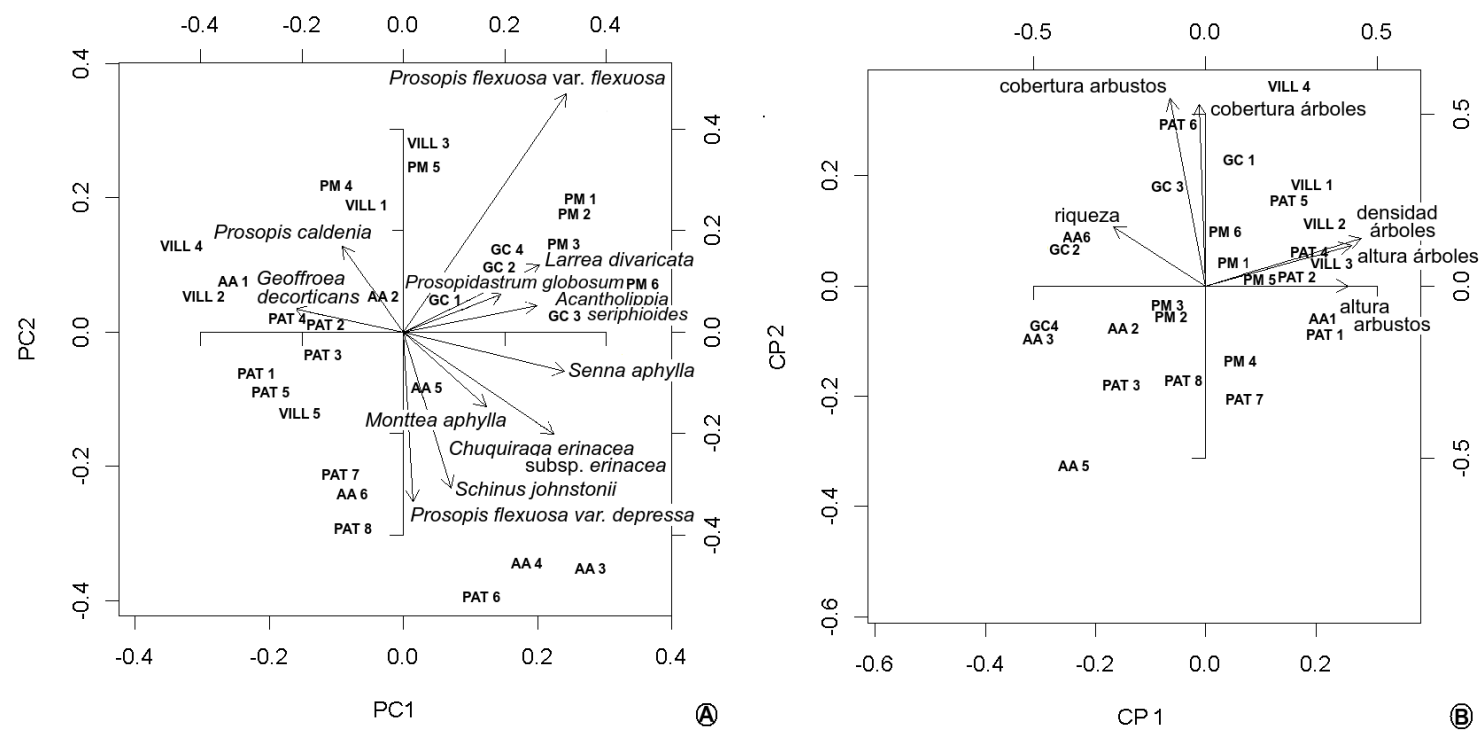

Fig. 2. Ordenamiento de los sitios sobre la base de la frecuencia de especies (A) y de las variables estructurales (B). CP: componente principal. AA: Adolfo Alsina. GC: General Conesa. PAT: Patagones. PM: Pichi Mahuida. VILL: Villarino. 
Tabla 1. Tendencias geográficas de las especies interpretadas a partir de la relación entre los ejes de ordenamiento y las coordenadas geográficas.

$\mathrm{O}$ : especies cuya frecuencia disminuye de oeste

a este, E: especies cuya frecuencia disminuye de este a oeste, $\mathrm{N}$, especies cuya frecuencia disminuye de norte a sur, S: especies cuya frecuencia disminuye de sur a norte, ST: especies frecuentes en toda el área estudiada y sin tendencias de variación geográfica.

\begin{tabular}{|lcc|}
\hline \multicolumn{1}{|c}{ Especie } & Dirección geográfica \\
\hline Senna aphylla & E-O & N-S \\
Acantholippia seriphioides & O & \\
Larrea divaricata & O & \\
Prosopidastrum globosum & O & \\
Monttea aphylla var. aphylla & O & \\
Prosopis flexuosa var. flexuosa & O & \\
Geoffroea decorticans & O & N \\
Prosopis caldenia & E & \\
Prosopis flexuosa var. depressa & & N \\
Schinus johnstonii & E & S \\
Cyclolepis genistoides & & S \\
Chuquiraga erinacea & & S \\
subsp. erinacea & & ST \\
Condalia microphylla & & ST \\
Lycium chilense & ST & \\
Ephedra triandra & ST & \\
\hline
\end{tabular}

y altura de árboles desde el extremo negativo hacia el positivo, mientras que la riqueza total de especies leñosas presentó una tendencia inversa (Fig. $1 \mathrm{~B}$ ). Este eje se correlacionó significativamente con la latitud $(r=-0,59 ; P<0,01)$ indicando una tendencia $\mathrm{S}-\mathrm{N}$ desde el extremo negativo hacia el positivo. Los sitios ubicados hacia el extremo negativo no presentaron componentes arbóreos, mientras que, en los que tendieron hacia el positivo, los valores correspondientes a la cobertura, densidad y altura de árboles variaron aproximadamente entre $10-30 \%$, 500-2000 árboles/ha y 2,5-3,5 m respectivamente (Fig. 2 B). El segundo eje reflejó un gradiente creciente de la riqueza total de especies leñosas y de la cobertura arbórea desde el extremo negativo hacia el positivo (Fig. 2 B). Este eje se correlacionó moderadamente con la longitud reflejando una tendencia E-O desde el extremo negativo hacia el positivo. Los sitios hacia el extremo negativo presentaron valores de riqueza y cobertura de arbustos de los sitios ubicados hacia el extremo inferior del eje 2, resultaron aproximadamente entre 4-6 especies y $15-40 \%$, mientras que en los ubicados hacia el extremo positivo esos valores fueron de entre 15-18 especies y 40-60\% (Fig. 2 B).

La variación de composición estuvo relacionada con la variación estructural ( $r$ de Mantel $=0,33$; $P<0,01$ ), pero ese valor se redujo cuando se controló el efecto de la distancia ( $r$ de Mantel parcial $=0,22 ; P<0,01)$.

\section{Discusión}

La variación de la composición florística estuvo claramente asociada con gradientes geográficos, indicando que los cambios más importantes ocurren en la dirección E-O, y en menor medida en dirección N-S. Los cambios estructurales resultaron menos asociados con la variación geográfica que los cambios composicionales, exhibiendo su mayor variación en dirección N-S, y en menor medida en la dirección E-O.

Los resultados de las pruebas de Mantel indican, en primer lugar, que la variación composicional está asociada con la variación estructural. Esa asociación se debe a que ambas características varían a lo largo de los gradientes geográficos N-S y E-O. Pero la prueba parcial de Mantel indicó una baja relación entre composición y estructura al controlar el efecto de la distancia entre sitios. Es decir, que las diferencias estructurales, no relacionadas con el gradiente geográfico, tienen poca relación con la composición. Las variaciones estructurales en sentido N-S reflejan la disminución en la participación de especies arbóreas en transición desde situaciones más propias del Espinal hasta el Monte. La variación E-O, en la que la estructura responde menos claramente al gradiente geográfico, representa un aumento de la cobertura de arbustos y de la riqueza total de especies leñosas. El sector E del área estudiada es el más afectado por actividades humanas relacionadas con la agricultura y ganadería (Arturi et. al., 2011). Las variaciones en la cobertura arbustiva y riqueza podrían diferenciar a las situaciones con distinta incidencia de los 
disturbios que, a su vez, coinciden parcialmente con el gradiente geográfico.

Las variaciones de la composición en sentido E-O muestran un aumento de la frecuencia de especies arbustivas como Senna aphylla, Acantholippia seriphioides, Larrea divaricata, Prosopidastrum globosum y Monttea aphylla, que son consideradas como especies típicas del Monte, así como especies acompañantes en el Espinal, Distrito del Caldén (sensu Cabrera, 1976). Por otra parte, las especies arbóreas típicas del Espinal, sensu Cabrera (1976), tendieron a reemplazarse desde el E (Prosopis caldenia y Geoffroea decorticans) hacia el $\mathrm{O}$ (Prosopis flexuosa var. flexuosa). En consecuencia, el gradiente de composición E-O desde el partido Villarino (Provincia de Buenos Aires) hasta el departamento de Pichi Mahuida (Provincia de Río Negro), al S del río Colorado no refleja claramente una transición Espinal-Monte. En cambio, el gradiente de composición asociado a la dirección $\mathrm{N}-\mathrm{S}$ indica una disminución de la frecuencia de especies arbóreas y un aumento de arbustos que, en este trabajo, se presentan como especies más típicas del Monte (Prosopis flexuosa var. depressa, Schinus johnstonii, Cyclolepis genistoides y Chuquiraga erinacea subsp. erinacea) sensu Cabrera, (1976). La variación fisonómica coincide con la interpretación florística, ya que la variación latitudinal estuvo mejor relacionada con las variables estructurales y reflejó que el estrato arbóreo de 3 a $4 \mathrm{~m}$ de altura existente en sitios del $\mathrm{N}$ del área estudiada, desapareció hacia el S. En este sentido, Roig et al. (2009) sugieren que las variaciones fisonómicas representan diferencias más claras entre el Monte y el Espinal que las variaciones florísticas.

Diferentes autores consideran que gran parte del área estudiada pertenece a la provincia fitogeográfica del Monte (León et al., 1998, Paruelo et al., 1992; Bran et al., 2000; SAyDS, 2002; 2005 y Roig et al., 2009) aunque diferencian el "Monte Oriental", al E de la provincia de Río Negro, en los departamentos Pichi Mahuida, General Conesa y Adolfo Alsina, del "Monte Austral típico" hacia el SO de Río Negro. El "Monte Oriental" puede incluso ser subdividido en "Monte Pampeano" (Bran et al., 2000; Roig et al., 2009), al E del departamento Pichi Mahuida, como una zona de "mezcla" con la provincia fitogeográfica del Espinal, caracterizada por la presencia de bosquetes de Prosopis caldenia, con precipitaciones entre 200 y $400 \mathrm{~mm}$ anuales, y
"Monte de Transición" (Bran et al., 2000) o "Monte Norpatagónico" (Roig et al., 2009), al O de Pichi Mahuida, NE de Avellaneda, General Conesa y Adolfo Alsina, con precipitaciones entre 150 y $250 \mathrm{~mm}$ anuales, caracterizados por la presencia de bosquetes de Prosopis flexuosa var. flexuosa y Geoffroea decorticans.

Las diferenciaciones propuestas por los autores Cabrera (1976); Cabrera \& Willink, (1980); Burkart et al., (1999); Brown et al., (2006); Morello et al., (2012) coinciden con las tendencias geográficas descriptas en este trabajo y que indican características vegetacionales más propias del Espinal en el $\mathrm{N}$ del área estudiada (Villarino, provincia de Buenos Aires y Pichi Mahuida y General Conesa, provincia de Río Negro) y un aumento de las características estructurales y composicionales más propias del Monte hacia el S (Patagones, provincia de Buenos Aires y Adolfo Alsina, provincia de Río Negro) en un amplio gradiente espacial asociado con variaciones climáticas. El efecto de los disturbios parece afectar en menor medida ese gradiente. Los resultados hallados sugieren que los disturbios promueven cambios más notables en la estructura que en la composición florística, de modo que resulta poco probable que provoquen un corrimiento de límites geográficos entre las ecorregiones del Espinal y el Monte. El mantenimiento de la composición a pesar del cambio estructural podría deberse a la capacidad de rebrote que presentan la mayoría de las especies leñosas de estas regiones, frente a la acción del fuego o daño mecánico (Bran et al., 2007; Kropfl et al., 2007; Peter et al., 2013). Este mecanismo permite la recuperación de la estructura a partir de los individuos preexistentes por lo que el cambio de composición estaría dado por la eventual mortalidad de una proporción de ellos dependiendo de la severidad del disturbio (Bran et al., 2007).

\section{Agradecimientos}

El trabajo fue financiado por la Agencia Nacional de Promoción Científica y Tecnológica y la Universidad Nacional de Río Negro (PICT 65/09, PICT-O 198/10, PI 40-C-222). Agradecemos a Raúl Herrera, Cintia Leder, Sabina Orsili, Belén Rodríguez y Martín Luna por el acompañamiento en las tareas de campo. 


\section{Bibliografía}

ANTON, A. M. \& F. O. ZULOAGA. 2014. Flora Argentina. Flora vascular de la república Argentina. Disponible en: http://www.floraargentina.edu.ar [Acceso Noviembre de 2014].

ARTURI, M. F. 2006. Situación Ambiental en la Ecorregión Espinal: 241-246. En: BROWN, A., U. MARTÍNEZ ORTIZ, M. ACERBI \& J. CORCUERA (eds.), La Situación Ambiental Argentina 2005. Fundación Vida Silvestre Argentina, Buenos Aires. $587 \mathrm{pp}$.

ARTURI, M. F.; S. S. TORRES ROBLES \& R. HERRERA SANTÁNGELO. 2011. Proceso de ordenación territorial de los recursos naturales renovables del Monte y Espinal de la provincia de Río Negro. $\mathrm{N}^{\circ}$ de inventario: 48845, reg: 10272. Consejo Federal de Inversiones (CFI), Ciudad Autónoma de Buenos Aires. Disponible en: http:// cfired.org.ar/Default.aspxmod $=$ databases $\&$ type $=2$ [Acceso: noviembre de 2014].

BRAN, D., J. A. AYESA \& C. LOPEZ. 2000. Regiones Ecológicas de Río Negro. Laboratorio de Teledetección-SIG INTA-EEA, Bariloche.

BRAN, D. E., G. A. CECCHI, J. J. GAITÁN, J. A. AYESA \& C. R. LÓPEZ. 2007. Efecto de la severidad de quemado sobre la regeneración de la vegetación en el Monte Austral. Ecología Austral 17:123-131.

BROWN, A., U. MARTÍNEZ ORTÍZ, M. ACERBI \& J. CORCUERA. 2006. La situación Ambiental Argentina. Fundación Vida Silvestre Argentina, Buenos Aires.

BURKART, R.; N. O. BÁRBARO; R. O. SÁNCHEZ \& D. A. GÓMEZ. 1999. Ecorregiones de la Argentina. Administración de Parques Nacionales, Buenos Aires.

CABRERA, A. L. 1976. Regiones fitogeográficas Argentinas. $2^{\circ}$ ed. Enc. Arg. Agricultura y Jardinería. Ed. ACME, S.A.I.C., Buenos Aires.

CABRERA, A. L. \& A. WILLINK, 1980. Biogeografía de América Latina. Serie Biología, Monografía 13. Organization of American States, Washington DC.

DIGBY, P. G. N. \& R. A. KEMPTON. 1987. Multivariate analysis of ecological communities. Chapman \& Hall, London.

FOSBERG, F. R. 1961. Classification of vegetation for general purposes. Trop. Ecol. 2:1-28.

GODAGNONE R. E. \& D. E. BRAN (EDS.). 2009. Inventario integrado de los recursos naturales de la provincia de Río Negro. Geología, hidrogeología, geomorfología, suelos, vegetación y fauna. Ediciones INTA, Buenos Aires.
HERNÁNDEZ BERMEJO, J. E \& H. SAINZ OLLERO. 1984. El análisis de semejanza aplicado al estudio de barreras y fronteras fitogeográficas: su aplicación a la corología y endemoflora ibéricas. Anales Jard. Bot. Madrid 40:421-432.

KRÖPFL, A. I, V. A. DEREGIBUS \& G. CECCHI, 2007. Disturbios en una estepa arbustiva del Monte: cambios en la vegetación. Ecología Austral 17: 257-268.

LEÓN, R. J. C., D. BRAN, M. COLLANTES, J. M. PARUELO \& A. SORIANO. 1998. Grandes unidades de la vegetación en la Patagonia extra andina. Ecología Austral 8:125-148.

MATEUCCI S. D. \& A. COLMA. 1982. Metodología para el estudio de la vegetación. Monografía $\mathrm{n}^{\circ}$ 22, Serie Biología. Secretaría General de la OEA, Washington D.C.

MORELLO, J., S. D. MATEUCCI, A. F. RODRÍGUEZ \& M. E. SILVA. 2012. Ecoregiones y complejos ecosistémicos argentinos $1^{\mathrm{a}}$ ed. Orientación Gráfica editora. Buenos Aires.

PARUELO, J. M., M. AGUIAR, R. A. GOLLUSCIO \& R. J. C. LEÓN. 1992. La patagonia extra andina: análisis de la estructura y el funcionamiento de la vegetación a distintas escalas. Ecología Austral 2: 123-136.

PETER, G., F. A. FUNK \& S. S. TORRES ROBLES. 2013. Responses of vegetation to different land-use histories involving grazing and fire in the North-east Patagonian Monte, Argentina. The Rangeland J. 35: 273-283.

R DEVELOPMENT CORE TEAM. 2009. R: A language and environment for statistical computing. $\mathrm{R}$ Foundation for Statistical Computing, Vienna, Austria. ISBN 3-900051-07-0. Disponible en: http:// www.R-project.org

ROIG, F. A., S. ROIG-JUÑENT \& V. CORBALÁN, 2009. Biogeography of the Monte Desert. J. Arid Env. 73: 164-172.

SAyDS. 2002. Atlas de los bosques nativos argentinos. BIRF-4085-AR, Buenos Aires.

SAyDS, 2005. Primer Inventario Nacional de Bosques Nativos. Proyecto Bosques Nativos y Áreas Protegidas. BIRF 4085-AR. Dirección de Bosques. Dirección Nacional de Recursos Naturales y Conservación de la Biodiversidad. Secretaría de Ambiente y Desarrollo Sustentable. Ministerio de Salud y Ambiente, Buenos Aires.

Recibido el 30 de noviembre de 2014, aceptado el 10 de abril de 2015. 
